\title{
Wavefront predictions for the automated assembly of optical systems
}

Schindlbeck, Christopher, Pape, Christian, Reithmeier, Eduard

Christopher Schindlbeck, Christian Pape, Eduard Reithmeier, "Wavefront predictions for the automated assembly of optical systems ," Proc. SPIE 10815, Optical Design and Testing VIII, 108150B (5 November 2018); doi: $10.1117 / 12.2500000$

SPIE. Event: SPIE/COS Photonics Asia, 2018, Beijing, China 


\title{
Wavefront predictions for the automated assembly of optical systems
}

\author{
Christopher Schindlbeck ${ }^{a}$, Christian Pape ${ }^{a}$, and Eduard Reithmeier ${ }^{a}$ \\ ${ }^{a}$ Leibniz Universität Hannover, Institute of Measurement and Automatic Control, \\ Nienburgerstr. 17, Hanover, Germany
}

\begin{abstract}
Industrial assembly of optical systems is still a tedious and cost-intensive task that is mostly dominated by manual labor. Positional fine-adjustment of optical components is pivotal to ensure a desired performance of the optical device at hand. In this paper, we use wavefront predictions to aim for fully automated assembly procedures. Wavefront measurements along with position identification methods can be utilized to continuously update a simulation model which in turn allows for predictions on future wavefront errors. This enables to take according correction measures during the assembly process if a certain wavefront tolerance specification is not met. In order to demonstrate the efficacy of the proposed approach and methods, a beam expander is sequentially assembled. The setup consists of a laser, two bi-convex lenses, and a Shack-Hartmann wavefront sensor and has to satisfy a certain wavefront tolerance specification after its assembly.
\end{abstract}

Keywords: Automated alignment, assembly, system identification, tolerancing, wavefronts, wavefront sensors, adaptive optics, beam expander

\section{INTRODUCTION}

Alignment of optical systems has been an ongoing research topic for decades. To realize large-scale production of optical systems, automated assembly is a key factor in increasing overall assembly speed within the manufacturing line and therefore lowering production costs.

To address this problem, a multitude of methods and approaches has been developed. For example, special freeform optical components can be machined ${ }^{1,2}$ to reduce the alignment problem. Since this transfers the production costs and time to the manufacturing of individual expensive freeform components, this cannot efficiently scale production throughput. Therefore, so-called active alignment try to place optical components to meet desired optical performance criteria.

Differential wavefront sensing ${ }^{3,4}$ is often used to align optical systems. For this, a quadrant photodetector is commonly employed and the misalignment is inferred by two overlaid light sources hitting the detector. Since this necessitates two beams, alternative methods have been developed to align optical system with only a single beam hitting the detector. With the help of a high-dimensional decomposition of the detector image space such as Zernike polynomials (and respective coefficients), several strategies ${ }^{5}$ for the alignment can be followed. Early works considered sensitivity tables ${ }^{6,7}$ to approach this problem but have been shown to perform poorly due to the nonlinear nature of Zernike polynomials and insufficiently close initial values. To account for this, nonlinear merit function ${ }^{8}$ optimization is typically employed. However, this does not account for positioning uncertainties and sensor noise and local minima may arise. More recent works increasingly utilize filtering such as the Extended Kalman Filter (EKF) in order to align optical systems such as to realize LED-based optical communication, ${ }^{9}$ wavefront reconstruction, ${ }^{10}$ or probabilistic approaches such as Bayesian exploration e.g. for the alignment of digital micro mirror devices. ${ }^{11}$

In this paper, we show how to improve wavefront predictions on future components to be assembled by following an EKF-based approach to improve the position identification. This can be effortlessly integrated into our previously developed predictor-corrector framework ${ }^{12}$ and results will be shown with experimental validation utilizing a beam expander as demonstrator.

Further author information:

E-mail: christopher.schindlbeck@imr.uni-hannover.de, Telephone: +49511 7623235

Optical Design and Testing VIII, edited by Yongtian Wang, Tina E. Kidger,

Kimio Tatsuno, Proc. of SPIE Vol. 10815, 108150B - C 2018 SPIE

CCC code: $0277-786 \mathrm{X} / 18 / \$ 18 \cdot$ doi: $10.1117 / 12.2500000$

Proc. of SPIE Vol. 10815 108150B-1 
This paper is structured as follows. Sec. 2 outlines three different approaches for the wavefront-based position identification which is essential to accurately predict future wavefronts in the assembly of optical systems. Sec. 3 summarizes the predictor-corrector framework of our previous work and the seamless integration of various identification methods. This lays the groundwork for the experiments presented in Sec. 4 and Sec. 5 concludes the paper.

\section{WAVEFRONT-BASED POSITION IDENTIFICATION}

For the predictions of future wavefronts, it is crucial to obtain an accurately identified optical model. In order to keep costs low for the manufacturing and assembly process of optical systems, it is desired to perform the identification with the sensors at hand and avoiding additional exteroceptive sensors to monitor the position of optical components or additional components such as beam splitters. The underlying problem can be formulated as such: Given an optical system with $n$ optical components and a detector with output $\mathbf{z}$, then an input light source maps to the output detector by

$$
\mathbf{z}=\mathbf{h}(\mathbf{X})
$$

where $\mathbf{h}$ is (in general) a nonlinear and surjective mapping of the set $\mathbf{X}$ of all optical components. Therein, each optical component $i \in\{1, \ldots, n\}$ is associated with a position vector $\mathbf{x}_{i} \in \mathbb{R}^{n_{d}}$ where $n_{d}$ are the degrees of freedom (DOF) of the optical components. The detector output needs to be given in vectorial form and should be a decomposition of the detector image. In this paper, we use the well-known Zernike polynomials to realize such decomposition.

Then, the problem formulation of the wavefront-based position identification consists of inferring the optical positions solely from detector measurements z. In this section, we discuss three approaches to solve the identification problem and compare them for the usability for sequential and automated optical assembly.

\section{Linear approach}

The most intuitive and computationally inexpensive approach to obtain optical component positions is to linearize (1), i.e.

$$
\frac{\partial \mathbf{z}}{\partial \mathbf{X}}=\frac{\partial \mathbf{h}(\mathbf{X})}{\partial \mathbf{X}} \approx S \Rightarrow \partial \mathbf{z}=S \cdot \partial \mathbf{X}
$$

where $S$ is the sensitivity matrix. This can be solved for positional displacement with the help of the pseudoinverse of the sensitivity matrix by $\partial \mathbf{X}=S^{\dagger} \cdot \partial \mathbf{z}$. As already mentioned in Sec. 1, the non-negligible nonlinearities of $\mathbf{z}$ as well as unknown initial values in general lead to poor identification quality.

2. Nonlinear approach

The most popular approach in current literature is to formulate a cost function that penalizes wavefront deviations caused by component misalignments. The cost function $J$, where additionally certain aberrations can be prioritized by introducing a weighting matrix ${ }^{13,14} \mathrm{~W}$, then reads as

$$
J(\mathbf{X} ; \mathbf{z}, W)=\frac{1}{2}\|\mathbf{h}(\mathbf{X})-\mathbf{z}\|_{W}^{2}=\frac{1}{2}(\mathbf{h}(\mathbf{X})-\mathbf{z})^{T} W(\mathbf{h}(\mathbf{X})-\mathbf{z})
$$

and can be minimized utilizing derivative-free or derivative-based optimization methods. If derivative-based optimization methods are chosen, the required gradient can be computed as

$$
\nabla_{\mathbf{X}} J(\mathbf{X} ; \mathbf{z}, W)=\left(\frac{\partial \mathbf{h}(\mathbf{X})}{\partial \mathbf{X}}\right)^{T} \frac{1}{2}\left(W+W^{T}\right)(\mathbf{h}(\mathbf{X})-\mathbf{z})=S^{T} \frac{1}{2}\left(W+W^{T}\right)(\mathbf{h}(\mathbf{X})-\mathbf{z})
$$

which simplifies for symmetric weighting matrices to

$$
\nabla_{\mathbf{X}} J(\mathbf{X} ; \mathbf{z}, W)=S^{T} W(\mathbf{h}(\mathbf{X})-\mathbf{z}) .
$$

The chosen optimization procedure then yields the identified optical positions at

$$
\mathbf{X}^{*}=\underset{\mathbf{X}}{\arg \min } J(\mathbf{X} ; \mathbf{z}, W) .
$$


Although this approach yields significantly better results than a linear approach since it accounts for the nonlinear nature of the mapping $\mathbf{h}$, still several drawbacks persist. Close initial values to the optimal solution are needed in order to avoid being stuck in local minima. Furthermore, optimization procedures will try to align components in the direction of the highest sensitivity.

3. Filtering approach

By augmenting equation (1) with the dynamics of a (controlled) positioning system, we get the discrete dynamics

$$
\begin{aligned}
\mathbf{x}_{k} & =\mathbf{A} \mathbf{x}_{k-1}+\mathbf{B} \mathbf{x}_{k-1, d}+\mathbf{w} \\
\mathbf{z}_{m, k} & =\mathbf{h}\left(\mathbf{x}_{k}\right)+\mathbf{v}
\end{aligned}
$$

with iteration step $k$ where $\mathbf{w}$ and $\mathbf{v}$ accounts for the positioning uncertainty and sensor noise, respectively. The transient response is not considered here since the time constants of the placement procedure are negligible compared to the overall assembly process. This allows to assume linear dynamics if the controller of the positioning system is tuned appropriately. $\mathbf{z}_{m}$ is the sensor measurement subject to noise and $\mathbf{x}_{d}$ the desired position commanded to the positioning system. Under the premise of being normally distributed with zero mean and covariance matrices $Q$ and $R$, i.e. $\mathbf{w} \sim \mathcal{N}(\mathbf{0}, Q)$ and $\mathbf{v} \sim \mathcal{N}(\mathbf{0}, R)$, an $\mathrm{EKF}^{15,16}$ can be utilized to estimate the actual position of the optical components. Since an EKF works with local linearization of the nonlinear equations, the sensitivity matrix of the linear approach can be utilized to linearize equation (5) locally. The equation (4) is already in linear form and the standard Kalman filter equations can be used without the necessity to linearize.

In conclusion, Tab. 1 shows how the three outlined approach compare to each other. In summary, for simulation and optical design it is still advisable to utilize the nonlinear approach since a simulation environment is free of uncertainties. However, for real-world identification where significant uncertainties of the positioning system as well as sensor noise arise, a filtering approach is more suited to obtain accurate position identification results.

\begin{tabular}{l|l|l} 
& Speed & Accuracy \\
\hline Linear & & \\
\hline Nonlinear & & \\
\hline Filtering & & \\
\hline
\end{tabular}

Table 1: Comparison of the three identification approaches with respect to speed and accuracy.

\section{PREDICTOR-CORRECTOR FRAMEWORK}

Previously, we proposed a predictor-corrector framework ${ }^{12}$ which will be shortly summarized in this section. We consider an optical system consisting of $n$ (static) optical components with a serial topology. The light source and detector are pre-aligned and therefore not considered for adjustment. We can distinguish between virtual positions $\mathbf{x}_{s}$ (provided by the optical simulation) and identified position (provided by the wavefrontbased position identification) $\mathbf{x}^{*}$. A unified notation comprising both entities is given by a hybrid position vector $\mathbf{X}_{H, i}=\left(\mathbf{X}_{i}^{*}, \mathbf{X}_{S, i}\right) \in \mathbb{R}^{n_{d} \cdot n}$, where $\mathbf{X}_{i}^{*}=\left(\mathbf{x}_{1}^{*}, \ldots, \mathbf{x}_{i}^{*}\right) \in \mathbb{R}^{n_{d} \cdot i}$ is the vector of the first $i$ identified optical component positions and $\mathbf{X}_{S, i}=\left(\mathbf{x}_{S, i+1}, \ldots ., \mathbf{x}_{S, n}\right) \in \mathbb{R}^{n_{d} \cdot(n-i)}$ is the complementary vector of the last $n-i$ virtual optical component positions. The index $i$ represents the current assembly step and therefore the configuration of the optical system (equaling the number of placed components).

\section{Workflow of Predictor-Corrector Framework}

1. Optical Component Placement

In this step, an optical component is placed into the real optical system at a position $\mathbf{x}_{i} \in \mathbf{X}_{H, i-1}$ given by the optical simulation. Simultaneously, this component is inserted in the simulation model. 


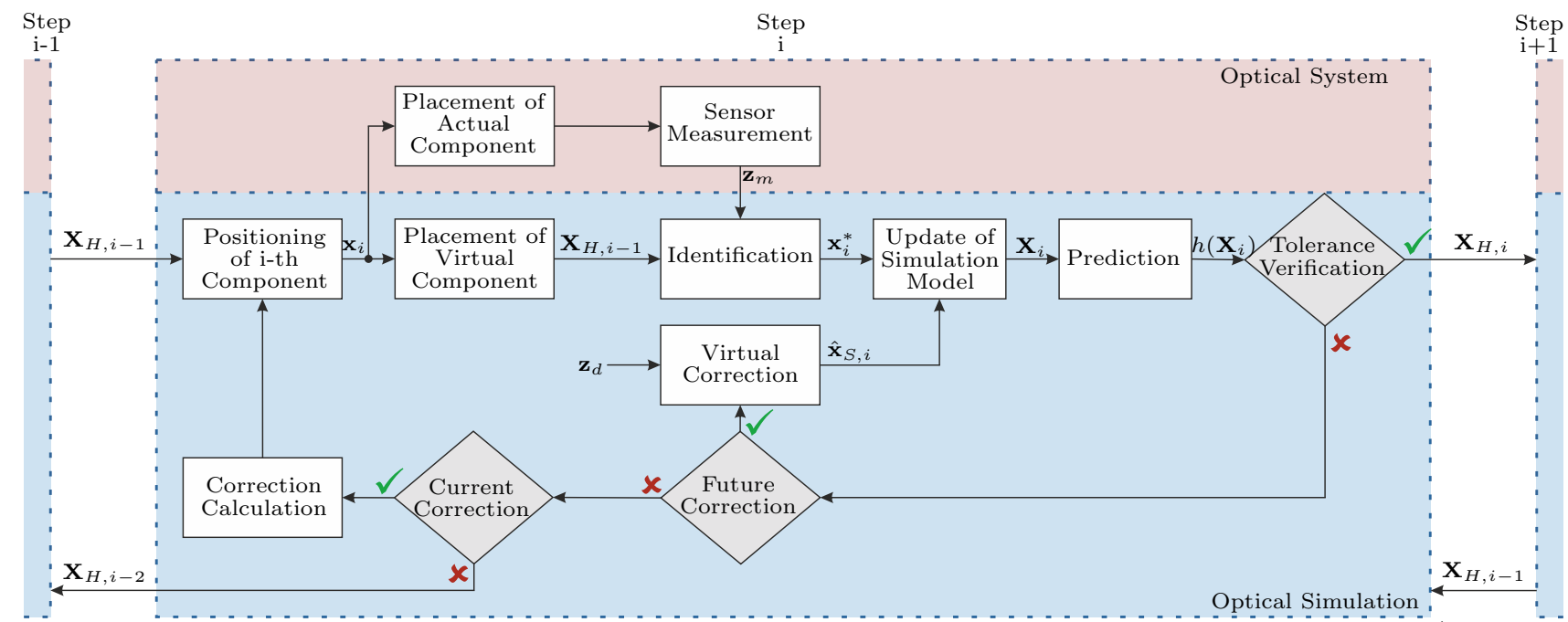

Figure 1: Predictor-corrector framework for the automated sequential assembly of optical systems (modified extension from previous work $\left.{ }^{12}\right)$.

\section{Sensor Measurement(s) and Identification}

Next, sensor measurements yield the current wavefront $\mathbf{z}_{m}$ at the detector. The current wavefront measurement can be used to update the simulation model. This corresponds to a position identification procedure which is described in Sec. 2. For filtering methods, multiple sensor measurements are required.

3. Model Update and Wavefront Prediction

After obtaining an updated simulation model from the identification results, the wavefront can be predicted by computing $\mathbf{z}_{p}=h\left(\mathbf{X}_{H, i}\right)$.

\section{Conformance Testing and Correction Calculation}

The predicted wavefront can analyzed w.r.t. certain criteria that the assembled optical system should comply with. Typically, a desired wavefront $\mathbf{z}_{d}$ is sought to be achieved after the assembly is completed. If the wavefront error deviates from a user-defined tolerance, according corrective measures need to be scrutinized and taken if possible. After having obtained an identified position vector $\mathbf{X}_{H, i}$, it is possible to predict the future wavefront $\mathbf{z}_{p}=h\left(\mathbf{X}_{H, i}\right)$ with the help of an optical simulation. This enables the verification of the predicted wavefront w.r.t. a user-defined tolerance threshold TOL. In this paper, the chosen metric is the weighted root-mean-square (RMS) error* such that the condition reads

$$
\left\|\mathbf{z}_{d}-\mathbf{z}_{p}\right\|_{W} \leq \text { TOL. }
$$

If (6) is violated, appropriate corrective measures need to be taken. First, it needs to be analyzed if a correction of future components can rectify this by computing the correction

$$
\hat{\mathbf{X}}_{S, i}=\underset{\mathbf{X}_{S, i} \subset \mathbf{X}_{H, i}}{\arg \min } J\left(\mathbf{X}_{H, i}, \mathbf{z}_{d}, W\right)
$$

and inserting it into

$$
\left\|\mathbf{z}_{d}-h\left(\mathbf{X}_{H, i}, \hat{\mathbf{X}}_{S, i}\right)\right\|_{W} \leq \text { TOL. }
$$

If (8) holds, we can proceed to the next assembly step. If not, the possibility of changing the position of the current optical component needs to be evaluated. The position correction can be computed in analogy to (7) and (8) by

$$
\hat{\mathbf{x}}_{i}=\underset{\mathbf{x}_{i} \in \mathbf{X}_{H, i}}{\arg \min } J\left(\mathbf{X}_{H, i}, \mathbf{z}_{d}, W\right)
$$

${ }^{*}$ This is different to the cost function (3) in the nonlinear approach where it leads to a simpler gradient for optimization purposes. The form chosen here is the more common RMS definition in literature to measure the wavefront error. 
and evaluate the corresponding predicted cost by

$$
\left\|\mathbf{z}_{d}-h\left(\mathbf{X}_{i-1}^{*}, \hat{\mathbf{x}}_{i}, \mathbf{X}_{S, i}\right)\right\|_{W} \leq \text { TOL. }
$$

Again, if (10) holds, the next assembly step is executed. If not, one has to go one step back in the assembly process and reiterate the process for $i \leftarrow i-1$.

\section{EXPERIMENTS}

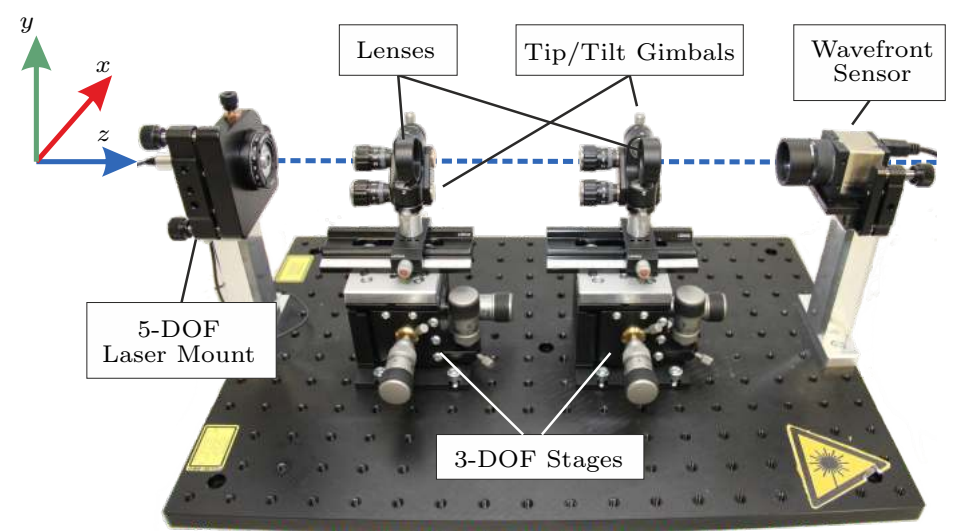

Figure 2: Experimental setup with two bi-convex lenses which are mounted inside 2-DOF gimbals fixated on 3 -DOF translational stages that are placed between a laser light source and a wavefront sensor.

Fig. 2 depicts the experimental setup which consists of a $532 \mathrm{~nm}$ laser light source, two bi-convex lenses (with $50 \mathrm{~mm}$ and $100 \mathrm{~mm}$ focal length, respectively), and a Shack-Hartmann wavefront sensor. The sensor provides the Zernike coefficients and in the experiments, we utilize the first ten Zernike coefficients according to the ZEMAX indexing scheme ${ }^{17}$ due to the usage of ZEMAX OpticStudio as simulation tool in this paper. The two lenses are placed inside gimbals that are able to adjust 2-DOF rotations and are each mounted on 3-DOF translational stages. The angular displacement can be realized in a $\pm 15^{\circ}$ range with a fine-adjustment of $1^{\circ}$ per revolution (and a resolution of 50 ticks). For the translational stages, a maximum travel of $\pm 2 \mathrm{~mm}$ can be made with a fine-adjustment of $50 \mu \mathrm{m}$ per revolution. To realize more displacement along the $z$-axis, the lenses are moved manually which in turn increases positioning uncertainty in this direction. This is done because sensitivity in this axis is low and therefore more displacement is necessary to improve identification accuracy. Based the aforementioned specifications, the covariance matrix for the combined positioning stages can be heuristically estimated as

$$
Q=\operatorname{diag}\left\{0.025 \mathrm{~mm}, 0.025 \mathrm{~mm}, 1 \mathrm{~mm}, 0.01^{\circ}, 0.01^{\circ}\right\} .
$$

Of course, this assumes that the coupling effects are negligible. For sensor noise identification, repeated measurements of the Zernike coefficients were obtained and a normal distribution can be fitted into the data. This results in the covariance matrix

$$
R=\operatorname{diag}\{0.0543,0.0276,0.0380,0.0145,0.0098,0.0150,0.0085,0.0096,0.0070,0.0086\}^{2}
$$

in wave units.

In this paper, we choose the RMS as wavefront criterion and the maximum tolerable wavefront error as $\lambda / 5$. Tab. 2 summarizes the experimental results for the two experiments that are carried out in this paper.

1. In the first experiment the system to be assembled should have a desired planar wavefront which corresponds to $\mathbf{z}=(0, \ldots, 0)^{T}$. Therefore, the lenses are at their nominal positions (w.r.t. the respective lens coordinate system) in the simulation model (see column for $\mathbf{X}_{H, 0}$ ). After inserting the first optical component, the position identification can be carried out which shows significant displacement from the nominal position and results therefore in a predicted cost that violates the given specification. However, by 
Table 2: Iterative assembly process for the beam expander in first and second experiment. Values for lens positions provided by initial design/simulation are shown in blue while values provided by identification/optical system are shown in red. Below is the corresponding predicted cost shown in green if tolerance requirement is met $(J / \lambda=0.2)$ and in red if violated.

\begin{tabular}{|c|c|c|c|c|c|c|c|c|c|}
\hline & \multicolumn{8}{|c|}{ First Experiment } \\
\hline & & $\begin{array}{c}\mathbf{X}_{H, 0}= \\
\left(\mathbf{x}_{S, 1}, \mathbf{x}_{S, 2}\right)\end{array}$ & $\begin{array}{r}\mathbf{X}_{H, 1}= \\
\left(\mathbf{x}_{1}^{*}, \mathbf{x}_{S, 2}\right)\end{array}$ & $\begin{array}{r}\mathbf{X}_{H, 1}= \\
\left(\mathbf{x}_{1}^{*}, \hat{\mathbf{x}}_{S, 2}\right)\end{array}$ & $\begin{array}{l}\mathbf{X}_{H, 2}= \\
\left(\mathbf{x}_{1}^{*}, \mathbf{x}_{2}^{*}\right)\end{array}$ & $\begin{array}{l}\mathbf{X}_{H, 2}= \\
\left(\mathbf{x}_{1}^{*}, \hat{\mathbf{x}}_{2}\right)\end{array}$ & $\begin{array}{l}\mathbf{X}_{H, 2}= \\
\left(\mathbf{x}_{1}^{*}, \mathbf{x}_{2}^{*}\right)\end{array}$ & $\begin{array}{l}\mathbf{X}_{H, 2}= \\
\left(\mathbf{x}_{1}^{*}, \hat{\mathbf{x}}_{2}\right)\end{array}$ & $\begin{array}{l}\mathbf{X}_{H, 2}= \\
\left(\mathbf{x}_{1}^{*}, \mathbf{x}_{2}^{*}\right)\end{array}$ \\
\hline \multicolumn{2}{|c|}{ Configuration } & 0 & \multicolumn{2}{|c|}{1} & \multicolumn{5}{|c|}{2} \\
\hline \multirow{5}{*}{ Lens 1} & $x / \mathrm{mm}$ & 0 & -0.8668 & - & - & - & - & - & - \\
\hline & $y / \mathrm{mm}$ & 0 & -0.4237 & - & - & - & - & - & - \\
\hline & $z / \mathrm{mm}$ & 0 & -8.2240 & - & - & - & - & - & - \\
\hline & $\theta_{x} /^{\circ}$ & 0 & -3.8063 & - & - & - & - & - & - \\
\hline & $\theta_{y} /{ }^{\circ}$ & 0 & 6.0565 & - & - & - & - & - & - \\
\hline \multirow{5}{*}{ Lens 2} & $x / \mathrm{mm}$ & 0 & 0 & -0.4910 & -0.6171 & -0.5451 & -0.6375 & -0.4929 & -0.4891 \\
\hline & $y / \mathrm{mm}$ & 0 & 0 & -0.1891 & -0.0793 & -0.1130 & -0.2346 & -0.1895 & -0.1831 \\
\hline & $z / \mathrm{mm}$ & 0 & 0 & -8.7163 & -15.8612 & -9.2058 & -8.8106 & -8.8116 & -9.1806 \\
\hline & $\theta_{x} /^{\circ}$ & 0 & 0 & -0.0640 & 1.8495 & 3.6507 & -0.0753 & -0.0763 & -0.0651 \\
\hline & $\theta_{y} /^{\circ}$ & 0 & 0 & 0.1714 & -0.5336 & 2.7236 & 0.2396 & -0.2426 & -0.1904 \\
\hline Pred. Cost & $J / \lambda$ & 0.0013 & 7.3299 & 0.0267 & 1.8299 & 0.0045 & 6.735 & 0.0278 & 0.1218 \\
\hline
\end{tabular}

\begin{tabular}{|c|c|c|c|c|c|c|c|c|c|}
\hline & \multicolumn{8}{|c|}{ Second Experiment } \\
\hline & & $\begin{array}{c}\mathbf{X}_{H, 0}= \\
\left(\mathbf{x}_{S, 1}, \mathbf{x}_{S, 2}\right)\end{array}$ & $\begin{array}{r}\mathbf{X}_{H, 1}= \\
\left(\mathbf{x}_{1}^{*}, \mathbf{x}_{S, 2}\right)\end{array}$ & $\begin{array}{r}\mathbf{X}_{H, 1}= \\
\left(\mathbf{x}_{1}^{*}, \hat{\mathbf{x}}_{S, 2}\right)\end{array}$ & $\begin{array}{l}\mathbf{X}_{H, 2}= \\
\left(\mathbf{x}_{1}^{*}, \mathbf{x}_{2}^{*}\right)\end{array}$ & $\begin{array}{l}\mathbf{X}_{H, 2}= \\
\left(\mathbf{x}_{1}^{*}, \hat{\mathbf{x}}_{2}\right)\end{array}$ & $\begin{array}{l}\mathbf{X}_{H, 2}= \\
\left(\mathbf{x}_{1}^{*}, \mathbf{x}_{2}^{*}\right)\end{array}$ & $\begin{array}{l}\mathbf{X}_{H, 2}= \\
\left(\mathbf{x}_{1}^{*}, \hat{\mathbf{x}}_{2}\right)\end{array}$ & $\begin{array}{l}\mathbf{X}_{H, 2}= \\
\left(\mathbf{x}_{1}^{*}, \mathbf{x}_{2}^{*}\right)\end{array}$ \\
\hline \multicolumn{2}{|c|}{ Configuration } & 0 & \multicolumn{2}{|c|}{1} & \multicolumn{5}{|c|}{2} \\
\hline \multirow{5}{*}{ Lens 1} & $x / \mathrm{mm}$ & 0 & -0.8990 & - & - & - & - & - & - \\
\hline & $y / \mathrm{mm}$ & 0 & -0.2935 & - & - & - & - & - & - \\
\hline & $z / \mathrm{mm}$ & 0 & -6.8386 & - & - & - & - & - & - \\
\hline & $\theta_{x} /^{\circ}$ & 0 & 0.0480 & - & - & _ & - & - & - \\
\hline & $\theta_{y} /^{\circ}$ & 0 & -0.14134 & - & - & - & - & - & - \\
\hline \multirow{5}{*}{ Lens 2} & $x / \mathrm{mm}$ & 0 & 0 & -0.9434 & -0.6171 & -0.9473 & -0.9210 & -0.9518 & -0.9496 \\
\hline & $y / \mathrm{mm}$ & 0 & 0 & -0.3081 & -0.1644 & -0.3092 & -0.3414 & -0.3112 & -0.3102 \\
\hline & $z / \mathrm{mm}$ & -5 & 0 & -11.9108 & -10.4847 & -11.9030 & -12.3483 & -11.8963 & -12.1051 \\
\hline & $\theta_{x} /^{\circ}$ & 0 & 0 & -0.0214 & -0.0645 & -0.0776 & -0.1755 & -0.0763 & -0.1709 \\
\hline & $\theta_{y} /^{\circ}$ & 0 & 0 & 0.0655 & 0.2280 & 0.2668 & 0.4975 & -0.2426 & 0.5038 \\
\hline Pred. Cost & $J / \lambda$ & 0 & 13.4221 & 0.0025 & 5.7785 & 0.0028 & 0.6175 & 0.0018 & 0.0649 \\
\hline
\end{tabular}

correcting the second lens in the simulation, it can be shown that this leads to maintaining the tolerance specification. Next, the second lens is placed in the optical train according to the new desired position $\hat{\mathbf{x}}_{S, 2}$. After two correction and identification steps, the desired tolerance of the optical system is maintained. A misalignment in $z$-direction of the first lens is compensated by shifting the second lens along the $z$-axis as well and the tip/tilt misalignment are compensated by translational corrections in $x$ - and $y$-axis. This can be attributed to the nonlinear approach for the computation of the correction terms.

2. In the second experiment, the desired wavefront to be achieved should have a convex shape (i.e. all Zernike coefficients are zero except for the defocus term which is about -0.3143). This corresponds to the same positions in the nominal design as in the first experiment expect for the second lens which is shifted $5 \mathrm{~mm}$ along the negative $z$-axis to achieve the desired wavefront. Similarly, an offset of the first lens of about $6 \mathrm{~mm}$ in $z$-direction can be seen after placing the first lens (see column for $\mathbf{X}_{H, 0}$ ) and the predictor-corrector framework tries to keep the $5 \mathrm{~mm}$ distance of the two lenses by correcting the position of the second lens by about $12 \mathrm{~mm}$ in negative $z$-direction (see column for $\mathbf{X}_{H, 1}=\left(\mathbf{x}_{1}^{*}, \hat{\mathbf{x}}_{S, 2}\right)$ ). Again, after two consecutive identification and current correction steps after the placement of the second lens, the specified tolerance requirement on the wavefront error is met. 


\section{CONCLUSION}

In this paper, we presented an approach to sequentially assemble and simultaneously align optical systems. During the assembly, wavefront predictions allow to forecast the tolerable error on the wavefront of the system to be assembled and therefore take according measures. For this, it is crucial to obtain the current positional state of the optical system. In order to avoid additional costs by external sensors, we aim at inferring the optical positions by solely the primary system sensor. To realize this, we outlined three approaches for the wavefront-based position identification problem: A computationally fast but inaccurate linear approach, a nonlinear approach yielding more accurate results but also involving higher computational costs, and a filtering approach taking uncertainties of the positioning system as well as the optical system into account. The last approach needs careful selection of trajectories as well as multiple sensor measurements but can lead to more accurate identification results. Experimental verification shows how to integrate the position identification methods into our previously developed predictor-corrector framework by assembling a two-lens system.

\section{REFERENCES}

1. Z. Li, X. Liu, F. Fang, X. Zhang, Z. Zeng, L. Zhu, and N. Yan, "Integrated manufacture of a freeform off-axis multi-reflective imaging system without optical alignment," Optics express 26(6), pp. 7625-7637, 2018.

2. Q. Meng, W. Wang, H. Ma, and J. Dong, "Easy-aligned off-axis three-mirror system with wide field of view using freeform surface based on integration of primary and tertiary mirror," Applied optics 53(14), pp. 3028-3034, 2014.

3. H. Lee, G. B. Dalton, I. A. Tosh, and S.-W. Kim, "Computer-guided alignment ii: Optical system alignment using differential wavefront sampling," Optics express 15(23), pp. 15424-15437, 2007.

4. H. Grote, G. Heinzel, A. Freise, S. Gossler, B. Willke, H. Lück, H. Ward, M. Casey, K. A. Strain, et al., "The automatic alignment system of geo 600," Classical and Quantum Gravity 19(7), p. 1849, 2002.

5. H. Lee, G. J. Hill, N. Drory, J. Ramsey, R. Bryant, and M. Shetrone, "Wavefront sensing for active alignment control of a telescope with dynamically varying pupil geometry: theory, implementation, on-sky performance," in Proc. SPIE, 10706, 2018.

6. J. W. Figoski, T. E. Shrode, and G. F. Moore, "Computer-aided alignment of a wide-field, three-mirror, unobscured, high-resolution sensor," in Recent trends in Optical systems design and computer lens design workshop II, 1049, pp. 166-178, SPIE, 1989.

7. J. Liu, F. Long, and W. Zhang, "Study on computer-aided alignment method," in Optical Design and Testing II, 5638, pp. 674-682, SPIE, 2005.

8. S. Kim, H.-S. Yang, Y.-W. Lee, and S.-W. Kim, "Merit function regression method for efficient alignment control of two-mirror optical systems," Optics Express 15(8), pp. 5059-5068, 2007.

9. P. B. Solanki, M. K. Al-Rubaiai, and X. Tan, "Extended kalman filter-based active alignment control for led optical communication," IEEE/ASME Transactions on Mechatronics , 2018.

10. J. Fang and D. Savransky, "Wavefront reconstruction with defocus and transverse shift estimation using kalman filtering," Optics and Lasers in Engineering 111, pp. 122-129, 2018.

11. K. B. Wynne, K. H. Knuth, and J. Petruccelli, "Enhanced optical alignment of a digital micro mirror device through bayesian adaptive exploration," AIP Advances 7(12), p. 125207, 2017.

12. C. Schindlbeck, C. Pape, and E. Reithmeier, "Predictor-corrector framework for the sequential assembly of optical systems based on wavefront sensing," Optics Express 26, pp. 10669-10681, 2018.

13. E. D. Kim, Y.-W. Choi, M.-S. Kang, and S. C. Choi, "Reverse-optimization alignment algorithm using zernike sensitivity," J. Opt. Soc. Korea 9, pp. 68-73, Jun 2005.

14. H.-S. Yang, S.-H. Kim, Y.-W. Lee, J.-B. Song, H.-G. Rhee, H.-Y. Lee, J.-H. Lee, I.-W. Lee, and S.-W. Kim, "Computer aided alignment using zernike coefficients," in Proc. of SPIE Vol. 6293, p. 62930I, 2006.

15. D. Simon, Optimal state estimation: Kalman, $H$ infinity, and nonlinear approaches, John Wiley \& Sons, 2006.

16. K. J. Keesman, System identification: an introduction, Springer Science \& Business Media, 2011.

17. V. L. Genberg, G. J. Michels, and K. B. Doyle, "Orthogonality of zernike polynomials," in Optomechanical Design and Engineering 2002, 4771, pp. 276-287, SPIE, 2002. 